Revista de Matemática: Teoría y Aplicaciones 2012 19(2) : 157-168

CIMPA - UCR ISSN: 1409-2433

\title{
MÉTODOS TIEMPO-FRECUENCIA BASADOS EN LA TRANSFORMADA WAVELET
}

\section{TIME-FREQUENCY METHODS BASED ON THE WAVELET TRANSFORM}

\author{
Eduardo P. SERrano* Marcela FABIO ${ }^{\dagger}$ \\ AlejAndRA Figliola $\ddagger$
}

Received: 3 Feb 2010; Revised: 10 Nov 2011; Accepted: 10 May 2012

\begin{abstract}
Resumen
La información contenida en una señal analógica se evidencia por su representación numérica. El par de Fourier en dos representaciones complementarias explicita las estructuras temporales y frecuenciales. Para detectar y caracterizar eventos que combinan esas estructuras en diversos y complejos patrones es necesario implementar métodos más refinados de representación conjunta tiempo-frecuencia.
\end{abstract}

*Centro de Matemática Aplicada, Universidad de San Martín, Martín de Irigoyen 3100, CP 1650 San Martín, Provincia de Buenos Aires, Argentina. E-Mail: eserrano@unsam.edu.ar

${ }^{\dagger}$ Misma dirección que/Same address as: E.P. Serrano. E-Mail: mfabio@unsam.edu.ar

${ }^{\ddagger}$ Instituto de Desarrollo Humano, Universidad de General Sarmiento, Juan María Gutierrez 1150, C.P. 1613 Los Polvorines, Provincia de Buenos Aires, Argentina. E-Mail: afigliol@ungs.edu.ar 
Entre diversas opciones, la transformada wavelet (en onditas $u$ ondaletas) brinda una eficiente herramienta tiempo-escala. Sus extensiones conducen a apropiadas representaciones conjuntas en un único contexto analítico y permiten implementar estrategias flexibles y bien adaptadas a las características de la señal.

Palabras clave: Representaciones tiempo-frecuencia, transformada wavelet, análisis de multirresolución, paquetes de wavelets, frecuencia instantánea.

\begin{abstract}
The information contained in an analog signal is reveled by its numerical representation. The Fourier pair, in two complementary representations, expounds time and frequency structures. However, to detect and characterize those events which combine both structures in diverse and complex patterns, it is necessary to implement more refined methods joint with appropriate time-frequency representations.

Among various options, the wavelet transform provides an efficient tool time-scale. Their extensions lead to appropriate representations coexisting in the same analytical context. Make it possible implement flexible strategies, well adapted to the characteristics of the signal.
\end{abstract}

Keywords: Time-frequency representations, wavelet transform, multiresolution analysis, wavelet packets, instantaneous frequency.

Mathematics Subject Classification: 42C40, 44A05.

\title{
1 Introducción
}

Una señal analógica es una función del tiempo $s(t), t \in \mathbb{R}$, que representa un fenómeno, un proceso o el comportamiento de un sistema. Relevar su información es el objetivo de su procesamiento. Pero tal información es polifacética y su interpretación depende del enfoque del análisis. Una cuestión central consiste, entonces, en contar con apropiadas técnicas de detección, caracterización e interpretación de los eventos y estructuras inmersos en la señal que, en su representación original, se mantienen enmascarados $u$ ocultos.

Suponemos en este trabajo que las señales son escalares, determinísticas y de energía finita, esto es, $s \in L^{2}(\mathbb{R})$.

En un primer examen podemos distinguir dos tipos de estructuras. La primera, temporal, es evidenciada por la representación original $s(t)$. Refleja eventos y propiedades localizados en torno de instantes o sobre 
intervalos temporales. La regularidad de la señal, sus cambios de comportamiento, sus valores extremos, su tendencia y otras propiedades análogas se visualizan explícitamente en la función representativa $s(t)$.

La segunda, frecuencial, está representada por la Transformada de Fourier $\widehat{s}(\omega)$. En este caso, la señal es interpretada como una superposición de ondas sinusoidales, periódicas de amplitud, frecuencia y fase constantes. Las ondas con amplitudes dominantes caracterizan las propiedades estacionarias y los patrones armónicos.

Por otra parte, cuando ellas se combinan, refuerzan o anulan entre sí, aparecen fenómenos oscilantes temporarios y locales o conforman ondas de amplitudes y espectro variable.

Estas representaciones se relacionan en el par de Fourier:

$$
\widehat{s}(\omega)=\frac{1}{\sqrt{2 \pi}} \int_{-\infty}^{\infty} s(t) e^{-\imath t \omega} d t ; s(t)=\frac{1}{\sqrt{2 \pi}} \int_{-\infty}^{\infty} \widehat{s}(\omega) e^{\imath t \omega} d \omega
$$

las cuales son expresiones complementarias, pero que no se asocian en una fórmula conjunta. Por lo tanto, por sí mismas, no permiten representar estructuras tiempo-frecuencia, como los eventos temporales oscilantes o las ondas no estacionarias con amplitudes y frecuencias que evolucionan en el tiempo.

Detectar y caracterizar este tipo de eventos presentes en muchos procesos y fenómenos naturales, requiere de refinamientos y representaciones apropiadas. Las mismas se denominan tiempo-frecuencia y pretenden expresar, de manera unificada, la relación entre los dos parámetros, [1], [3], [5].

\section{Representación tiempo-frecuencia}

Consideremos una señal $s \in L^{2}(\mathbb{R})$, normalizada, el par de densidades dadas por el par de Fourier: $\left(|s(t)|^{2} ;|\widehat{s}(\omega)|^{2}\right)$ y sus repectivos desvíos estándar $\sigma_{t}, \sigma_{\omega}$. Se dice que la señal es localizada en el dominio tiempofrecuencia si $\sigma_{t} \sigma_{\omega}<+\infty$.

Esta condición nos abre un espacio común donde pretendemos describir la distribución de la energía en forma conjunta. Sin embargo, tal representación conjunta no se deduce en forma automática del par de Fourier y la decisión no es trivial. El principio de incertidumbre de Heisemberg $\sigma_{t} \sigma_{\omega} \geq H$, cota universal, expresa la imposibilidad de representar formalmente la relación tiempo-frecuencia en vecindades de radio arbitrariamente pequeño e indica que las densidades sólo pueden relacionarse con precisiones en inversa proporción. En consecuencia, descartada una relación conjunta ideal, existe una amplia gama de criterios para aproximarla [3], [5]. 
Denotemos $T_{\lambda}$ y $\Omega_{\lambda}$ intervalos abiertos, temporal y frecuencial. Un cubo $Q_{\lambda}=T_{\lambda} \times \Omega_{\lambda}$, en el dominio tiempo-frecuencia, se dice elemental si existe una función normalizada o átomo tiempo-frecuencia $\phi_{\lambda}$ asociado tal que:

$$
\int_{t \in T_{\lambda}}\left|\phi_{\lambda}(t)\right|^{2} d t=1-\epsilon=\int_{|\omega| \in \Omega_{\lambda}}\left|\widehat{\phi}_{\lambda}(\omega)\right|^{2} d \omega
$$

para cierto $\epsilon>0$ pequeño y la medida $\left|Q_{\lambda}\right|=\left|T_{\lambda}\right|\left|\Omega_{\lambda}\right|$ cercana a la cota de Heisemberg. La información temporal y frecuencial del átomo se concentra en los respectivos intervalos.

En nuestro enfoque, supondremos que la energía conjunta de la señal está soportada en una unión de cubos elementales $\bigcup_{\lambda \in \Lambda} Q_{\lambda}$, asociada a una familia de átomos $\left\{\phi_{\lambda}, \lambda \in \Lambda\right\}$ y se asigna a cada cubo la energía $E_{\lambda}(s)=$ $\left|<s, \phi_{\lambda}>\right|^{2}$. Entonces, decimos que el conjunto $\left\{\left(Q_{\lambda}, \phi_{\lambda}, E_{\lambda}(s)\right) ; \lambda \in \Lambda\right\}$ constituye una representación tiempo-frecuencia de la señal.

La cuestión esencial consiste en la elección de los cubos y átomos asociados. ¿Qué propiedades debemos requerir a la representación?

En principio, se definen las aproximaciones locales sobre cada cubo elemental como $s_{\lambda}(t)=<s, \phi_{\lambda}>\phi_{\lambda}(t)$ y $\widehat{s}_{\lambda}(\omega)=<s, \phi_{\lambda}>\widehat{\phi}_{\lambda}(\omega)$ y la densidad conjunta local:

$$
S_{\lambda}(t, \omega)=\left|<s, \phi_{\lambda}>\right|^{2}\left|\phi_{\lambda}(t)\right|^{2}\left|\widehat{\phi}_{\lambda}(\omega)\right|^{2}
$$

que, integrada en ambos parámetros, nos devuelve la energía $E_{\lambda}(s)$.

A partir de estas definiciones formulamos los siguientes requerimientos:

- que exista una fórmula integral o de suma, respecto del parámetro $\lambda$, según su dominio sea continuo o discreto, estable, que permita reconstruir la señal,

- que mediante la reconstrucción o síntesis parcial puedan aislarse eventos significativos o de particular interés, como partes componentes separables,

- que por medio de la integración o suma respecto del parámetro $\lambda$ de las densidades locales $S_{\lambda}(t, \omega)$ quede definida una densidad conjunta $S(t, \omega)$ no negativa, tal que:

$$
\int_{-\infty}^{\infty} \int_{-\infty}^{\infty} S(t, \omega) d t d \omega=\|s\|^{2}
$$

En tal caso, $S(t, \omega)$ proporciona un espectro conjunto que describe la distribución de la energía de la señal localizada en el dominio tiempo-frecuencia, 
- que la medida del soporte $\left|\bigcup_{\lambda \in \Lambda} Q_{\lambda}\right|$ sea minimal, lo cual asegura que la representación sea precisa,

- que la entropía de la representación sea baja, es decir, que la información relevante se concentre en un dominio $\Lambda$ de medida o cardinal relativamente pequeño.

¿Es posible equilibrar estos requerimientos? ¿Cómo diseñar la familia de cubos o de átomos? Generalmente, la representación está asociada con transformadas integrales que reorganizan la información de la señal modificando el enfoque o resaltando estructuras de interés.

Por otra parte, los átomos pueden agruparse en familias de funciones, con estructuras o propiedades similares, constituyendo bibiliotecas de bases o de marcos. A partir de estas bibliotecas, la estrategia de diseño puede adaptarse a las particularidades de la señal y del problema por abordar, [2], [6]. Enfocaremos nuestra atención sobre alternativas, recalando luego en las basadas en la Transformada Wavelet, [5], [2], [9].

Más allá de nuestro enfoque, existen otras metodologías de representación, pero el tratamiento de este tema está más allá del horizonte del trabajo. Referimos a la bibliografía de referencia para mayores detalles, $[1],[3],[5],[6]$.

\section{Transformada de Gabor}

Una primera alternativa consiste en localizar la Transformada de Fourier mediante la acción de apropiadas ventanas. Se escanea la señal con una ventana modulada deslizante mientras se calcula el espectro local.

Entre varias posibilidades, la ventana puede ser una función gaussiana. Para cierto $\alpha>0$, fijo, se define la ventana $g_{\alpha}(t)=\frac{1}{2 \sqrt{\alpha \pi}} e^{-\frac{t^{2}}{4 \alpha}} \mathrm{y}$, para cada $s \in L^{2}(\mathbb{R}), \alpha>0, b \in \mathbb{R}$, la transformada de Gabor es:

$$
\left(G_{b}^{\alpha} s\right)(\omega)=\int_{-\infty}^{\infty} e^{-i \omega t} s(t) g_{\alpha}(t-b) d t .
$$

La señal puede sintetizarse a partir de la fórmula

$$
\widehat{s}(\omega)=\int_{-\infty}^{\infty}\left(G_{b}^{\alpha} s\right)(\omega) d b
$$

entendida en el sentido de $L^{2}(\mathbb{R})$.

Observemos que, de esta forma, la señal resulta de la superposición de los átomos de Gabor $G_{b, \omega}^{\alpha}(t)=e^{i \omega t} g_{\alpha}(t-b)$ con $b, \omega \in \mathbb{R}$ y que $\left(G_{b}^{\alpha} s\right)(\omega)=<s, G_{b, \omega}^{\alpha}>$. 
Los átomos tienen un ancho de banda constante, igual a $2 \sqrt{\alpha}$ y están localizados en los cubos $[b-\sqrt{\alpha}, b+\sqrt{\alpha}] \times\left[\omega-\frac{1}{2 \sqrt{\alpha}}, \omega+\frac{1}{2 \sqrt{\alpha}}\right]$.

La precisión tiempo-frecuencia es fija, dependiente del parámetro $\alpha$ y es ésta una de las principales limitaciones de la transformada, ya que, normalmente, los eventos asociados a las altas frecuencias poseen duración relativamente breve y viceversa. Por lo tanto, la eventual convivencia entre tales componentes puede no ser detectada.

Por otra parte, es posible tomar familias discretas $\left\{G_{b_{k}, \omega_{n}}\right\}$, definir la correspondiente transformada discreta y representar la señal por una serie. Pero tales familias, cuando la ventana está bien localizada, no pueden constituir bases ortonormales. Sólo son admisibles marcos, sobre-completos, sin unicidad de representación, [5], [9].

\section{Bases trigonométricas locales}

Otra alternativa consiste en localizar el análisis de Fourier sobre un cubrimiento de intervalos del dominio temporal. En cada intervalo la señal se suaviza con una ventana y se descompone en una serie de Fourier.

Los átomos son senos o cosenos de frecuencia definida y afectados por ventanas. Pero ahora, a diferencia de Gabor, las formas de onda y anchos de banda y, por lo tanto, los espectros locales pueden decidirse con amplio grado de libertad. Más aún es posible organizar los átomos en una generosa biblioteca de bases ortonormales trigonométricas locales.

Estas exigencias suponen técnicas refinadas para el diseño de las particiones y las ventanas. Referimos a [2], [5] para los detalles. Ilustramos con una clásica alternativa, las denominadas Bases de Malvar-Wilson.

El cubrimiento por intervalos se define a partir de una secuencia monótona y arbitraria de nodos $\mathbb{R}=\bigcup_{k}\left[t_{k}, t_{k+1}\right]$. Sobre cada intervalo ampliado $\left[t_{k}-\alpha_{k}, t_{k+1}+\alpha_{k+1}\right]$, donde $\alpha_{k}+\alpha_{k+1} \leq t_{k+1}-t_{k}$, se define un ventana $w_{k}$ con precisas propiedades.

Estas ventanas se superponen parcialmente y definen un perfil de onda sobre cada intervalo. Entonces, los átomos se definen como:

$$
u_{k, n}=\sqrt{\frac{2}{T_{k}}} w_{k}(t) \cos \left[\frac{\pi}{T_{k}}(n+1 / 2)\left(t-t_{k}\right)\right],
$$

donde $T_{k}=t_{k+1}-t_{k}, n=1,2, \ldots$ y $k \in \mathbb{Z}$. Bajo las condiciones de diseño esta colección constituye una base ortonormal.

Es interesante observar que, de acuerdo al diseño, las bases pueden modificarse intercalando o suprimiendo nodos en la partición, lo cual facilita la implementación de algoritmos de mejora y de búsqueda de una 
base óptima, respecto de algún criterio, de la mínima entropía de la representación.

Esta estructura de biblioteca organizada, que posibilita eficientes algoritmos de mejora, puede aplicarse a otras familias de átomos, particularmente a los paquetes de wavelets, como luego expondremos.

Merece destacarse también que la representación en término de bases de Malvar puede escribirse como:

$$
s(t)=\sum_{k, n}<s, u_{k, n}>u_{k, n}(t)=\sum_{k, n} A_{k, n}(t) \cos \left(\omega_{k, n}(t)\right),
$$

siendo $\omega_{k, n}(t)$ una función afin de $t$, lo que sugiere otras extensiones, que comentamos en la sección siguiente.

Las bases trigonométricas locales poseen importantes aplicaciones, pero la segmentación del dominio temporal seguido del clásico análisis de Fourier no permite caracterizar eventos autosimilares o donde la escala juega un papel relevante, $[6]$.

\section{Chirplets}

La fórmula (8) puede generalizarse en la representación:

$$
s(t)=\sum_{\lambda} A_{\lambda}(t) \cos \left(\omega_{\lambda}(t)\right)
$$

donde las funciones $\omega_{\lambda}(t)$ no son necesariamente lineales. En tal caso, estas series de Fourier generalizadas permiten representar estructuras complejas con espectros y amplitudes variando en el tiempo.

Gran número de alternativas se abren en esta dirección. En general, si las funciones $\omega_{\lambda}(t)$ son diferenciables y las amplitudes varían en forma relativamente suave, la representación suele denominarse multicomponente $A M-F M$ y para cada átomo $\cos \left(\omega_{\lambda}(t)\right)$ está definida la frecuencia instantánea $\left|\omega_{\lambda}^{\prime}(t)\right|$ que puede calcularse vía la transformada de Hilbert, $[5]$.

Una interesente técnica empírica, de descomposición en término de ondas o funciones modales intrínsecas es la denominada Transformada de Hilbert- Huang, [4]. En este caso, los átomos son ondas de frecuencia casi constante y la amplitud es variable.

Cuando las frecuencias varían en forma no lineal - cuadrática o racional, por ejemplo - los átomos suelen denominarse chirplets. Las representaciones están asociadas a diversas transformadas integrales o, en especiales casos, a familias de bases o marcos. 
Como ilustración, mencionemos las bases de Wilson-Malvar moduladas, extensión de las trigonométricas. Se basa en los átomos modulados:

$$
u_{k, n}=\sqrt{\frac{2}{T_{k}}} e^{i\left(a t^{2} / 2+b t\right)} w_{k}(t) \sin \left[\frac{\pi}{T_{k}}(n+1 / 2)\left(t-t_{k}\right)\right]
$$

para cada intervalo $\left[t_{k}, t_{k+1}\right]$ y donde $T_{k}=t_{k+1}-t_{k}, n=1,2, \ldots$ y $k \in \mathbb{Z}$. Bajo las condiciones de diseño antes mencionada, esta colección constituye una base ortonormal, [2].

\section{Transformada Wavelet}

Desde el punto de vista de Gabor apreciamos que para analizar señales donde conviven componentes de alta y bajas frecuencias es necesario contar con representaciones tiempo-frecuencia más flexibles y obtener métodos que permitan el ensanchamiento o estrechamiento de las ventanas temporales para permitir el reconocimiento de las bajas o altas frecuencias, respectivamente.

Por otra parte, la idea sugerida por los chirplets, de contar con átomos modulados en frecuencia, es también atractiva para atender relaciones de autosimilaridad o separar eventos por rangos de frecuencias. Esta ideas se combinan en la Transformada Wavelet. Los átomos se generan mediante la acción de los operadores de escala y de traslación sobre una única función o wavelet madre.

Partimos de una wavelet básica $\psi \in L^{2}(\mathbb{R})$, oscilante, centrada en una vecindad de $t=1 / 2$, normalizada y verificando cierta condición de admisibilidad $c_{\psi}<+\infty,[2],[5],[9]$.

Si se modifican sus oscilaciones y se la desplaza en el tiempo, se obtiene la familia $\left\{\psi_{a, b}(t)=\frac{1}{\sqrt{|a|}} \psi\left(\frac{t-b}{a}\right)\right\}$ con $b$ y $a$ parámetros de traslación y dilatación, respectivamente.

Se define la correspondiente transformada wavelet continua (CWT) de $s \in L^{2}(\mathbb{R})$ como:

$$
\mathcal{W} s(b, a)=<s, \psi_{b, a}>=\int_{-\infty}^{\infty} s(t) \psi_{a, b}^{*}(t) d t
$$

Observemos que las modulaciones de Gabor han sido reemplazadas por dilataciones. El parámetro $a \in \mathbb{R}-\{0\}$ es el de dilatación o escala y el $b \in \mathbb{R}$ es el de desplazamiento. Si la wavelet madre es una función real, basta tomar $a>0$.

Bajo estas condiciones es válida la fórmula de inversión:

$$
s(t)=\frac{1}{c_{\psi}} \int_{-\infty}^{\infty} \int_{-\infty}^{\infty} \mathcal{W} s(b, a) \psi_{a, b}(t) d b \frac{d a}{a^{2}} .
$$


Suponiendo que la wavelet es real, y que $|\psi(t)|$ está concentrada en el intervalo $\left[1 / 2-\sigma_{\psi}, 1 / 2+\sigma_{\psi}\right]$ y $|\widehat{\psi}(\omega)|$ está concentrada en la banda $\left[3 \pi / 2-\sigma_{\widehat{\psi}}, 3 \pi / 2+\sigma_{\widehat{\psi}}\right]$, la localización en el plano tiempo-frecuencia se concentra en los cubos, de medida $4 \sigma_{\psi} \sigma_{\widehat{\psi}}$, constante, $\left[b+a\left(1 / 2-\sigma_{\psi}\right), b+\right.$ $\left.a\left(1 / 2+\sigma_{\psi}\right)\right] \times\left[\left(3 \pi / 2-\sigma_{\widehat{\psi}}\right) / a,\left(3 \pi /(2)+\sigma_{\widehat{\psi}}\right) / a\right]$.

Las oscilaciones de la wavelet se concentran para valores de $a \rightarrow 0$ y se dilatan para valores de $a \rightarrow+\infty$. Vale la pena remarcar que éste no es un cambio de frecuencia sino de escala. Como en la caso de Gabor, se escanea la señal mientras se calcula la transformada y la información tiempo-escala se va revelando. Por esta razón, su aplicación posee eficiencia óptima para la detección y caracterización de eventos de duración limitada asociados con rangos de frecuencias con precisión cercana al límite de Heisemberg.

La transformada continua es consistente con los desplazamientos o modulaciones de la señal, particularmente con las relaciones de autosimilaridad. Sin embargo, en la práctica los conjuntos de parámetros se discretizan. En primer lugar, si el parámetro de escala se restringe como $a_{j}=2^{-j}$, se obtiene la transformada semi-discreta o diádica. Esto implica una partición diádica o por escalas del dominio frecuencial, en un esquema del tipo Littlewood-Paley, manteniendo la invariancia respecto del operador de traslación. Estas propiedades la hacen sumamente útil para algunas aplicaciones, como el estudio de la turbulencia o la caracterización de la regularidad local, [6].

Para muy especiales elecciones de la wavelet $\psi$ y la red discreta de parámetros $a_{j}=2^{-j}$ y $b_{j k}=2^{j} k$, con $k \in \mathbb{Z}$, la familia $\left\{\psi_{j k}(t)=\right.$ $\left.2^{j / 2} \psi\left(2^{j} t-k\right)\right\}$ constituye una base ortogonal del espacio de señales de finita energía.

Entonces, los coeficientes atómicos $c_{j k}(s)$ constituyen la transformada wavelet discreta (DWT), ortogonal y se tiene la descomposición atómica:

$$
s(t)=\sum_{j} \sum_{k} c_{j k}(s) \psi_{j k}(t)=\sum_{j} q_{j}(s)(t) .
$$

Cada componente $q_{j}(s)(t)$ corresponde a una escala u octava y es la proyección ortogonal de $s(t)$ sobre un subespacio wavelet $\mathcal{W}_{j}$. En correspondencia con una wavelet ortogonal, estos subespacios son mutuamente ortogonales y su suma directa reconstuye el espacio de señales. Por otra parte, las sumas parciales $\mathcal{V}_{j}=\oplus_{h<j} \mathcal{W}_{h}=\mathcal{V}_{j-1} \oplus \mathcal{W}_{j-1}$ definen los espacios de escala. Los mismos se relacionan en una particular estructura denominada Análisis de Multirresolución, (AMR), [2], [6], [9].

En este contexto es posible la descomposición sucesiva de la señal mediante un algoritimo recursivo de Mallat, el cual se realiza por sucesivas convoluciones discretas, a partir de los datos de $s(t)$, con un par de filtros 
digitales $F$ y $G$ pasa-bajo y pasa-banda, respectivamente. Estos filtros dependen de la wavelet madre utilizada. En el caso ortogonal, la aplicación del algoritrmo con los mismos filtros permite sintetizar la señal $s(t)$, [5].

La propiedad de ortogonalidad, tanto de las wavelets como de los subespacios $\mathcal{W}_{j}$, puede relajarse y el par $(F, G)$ reemplazarse por dos pares de filtros, $(F, G)$ de análisis y $(\tilde{F}, \tilde{G})$ de síntesis, respectivamente, [5], [9].

\section{Paquetes de wavelets}

Una wavelet ortogonal $\psi$, suave y con decaimiento exponencial, posee una transformada de Fourier de la forma $\widehat{\psi}(\omega)=|\widehat{\psi}(\omega)| e^{-\imath \omega / 2}$ concentrada en la banda $\pi / 2 \leq|\omega| \leq 4 \pi$. Sin embargo, la wavelet no tiene una frecuencia definida ya que $|\widehat{\psi}|$ es casi uniforme en $\pi \leq|\omega| \leq 2 \pi$, [5]. Por esta razón, los coeficientes atómicos $c_{j k}(s)$ no especifican frecuencias en el rango y, en cierto sentido, enmascaran las componentes oscilantes, los patrones armónicos o las ondas monocromáticas presentes en la señal. Para detectar y caracterizar tales eventos es necesario profundizar el análisis o retornar a los esquemas de Gabor o las bases trigonométricas locales, [2].

La idea es realizar este propósito en el contexto del análisis wavelet, más precisamente en el esquema de multirresolución, de modo de unificar el marco del análisis, [7].

Los paquetes de wavelets proporcionan una chance para tales propósitos. Son nuevas funciones elementales, que extienden la familia de wavelets y poseen una mejor localización en frecuencia.

$\mathrm{Su}$ principal aporte es que tales funciones pueden organizarse en bibliotecas de bases o de marcos del espacio $L^{2}(\mathbb{R})$ y se asocian a algoritmos eficientes para calcular los respectivos coeficientes de representación y para seleccionar un base óptima.

Existen diversas alternativas para tales propósitos. Los paquetes del tipo Coifman, Meyer y Wickerhauser iteran la acción de los filtros conjugados $F$ y $G$ y generan un tipo particular de funciones elementales, teóricamente bien localizadas en frecuencia. En la práctica, las iteraciones generan indeseables artefactos por lo cual su aplicación es limitada, [2].

Otro enfoque posible consiste en generar, en el contexto de un análisis multirresolución, apropiadas funciones elementales, casi-monocromáticas de duración limitada, con una frecuencia instantánea característica.

En [7] los autores han desarrollado este tipo de funciones, partiendo de proyecciones locales de las funciones trigonométricas sobre los espacios $\mathcal{W}_{j}$. Por tal razón son denominadas paquetes de wavelets trigonométricos. En [7] y [8] pueden verse los detalles del diseño y sus fundamentos.

Para cada entero $m \geq 1$, se definen las frecuencias fundamentales 
$\omega_{m h}=\pi+\frac{2 h \pi}{2^{m}}$ con $0 \leq h \leq 2^{m-1}$ y apropiadas matrices ortogonales de Fourier, $\left(F_{m}(l, k)\right)_{0 \leq l, k \leq 2^{m}-1}$, de dimensión $2^{m}$.

La clave consiste en adecuar pesos positivos $\mu_{m k} \mathrm{y}$ definir las funciones básicas en cada espacio wavelet $\mathcal{W}_{j}$ en la forma:

$$
\theta_{m, j, l, n}(t)=\sum_{k=0}^{2^{m}-1} \mu_{m k} F_{m}(l, k) \psi_{j, n+k}(t) .
$$

El parámetro $m$ puede decidirse de forma independiente para cada subespacio $\mathcal{W}_{j}$. Las funciones $\theta_{m, j, l, n}(t)$ se asemejan a senos o cosenos modulados por una ventana casi gaussiana y su transformada está bien localizada en torno de una frecuencia fundamental $2^{j} \omega_{m h}$. Esta propiedad de localización y las formas de onda depende de la longitud de filtros $F_{m}(l, k)$ y de los pesos $\mu_{m k}$.

Dado que las traslaciones de las funciones según el índice $n$ superponen parcialmente $2^{m}$ ventanas sobre cada punto, el análisis con este tipo de paquetes se asemeja a un esquema del tipo Gabor por escalas, en cada subespacio $\mathcal{W}_{j}$.

Bajo las condiciones del diseño cada familia $\left\{\theta_{m, j, l, n}\right\}$ es sobre completa, pero constituye un marco de Parseval de $\mathcal{W}_{j}$ [7]. Por lo tanto, las proyecciones $q_{j}(s) \in \mathcal{W}_{j}$ se representan eficientemente en la forma:

$$
q_{j}(t)=\sum_{n=-\infty}^{+\infty} \sum_{l=0}^{2^{m}-1}<q_{j}(s), \theta_{m, j, l, n}>\theta_{m, j, l, n}(t) .
$$

Los coeficientes de la representación se calculan convolucionando los coeficientes $c_{j k}(s)$ con los filtros $\mu_{m k} F_{m}(l, k)$.

La utilización de las herramientas presentadas en este artículo facilita una eficiente representación de la señal como superposición de ondas asociadas a una frecuencia definida, con una frecuencia instantánea específica y amplitud variando el tiempo, en forma análoga a las funciones modales intrísecas. Esta representación aproxima la estructura de la fórmula (9).

Este diseño puede extenderse a una familia de paquetes analíticos, a partir de la transformada de Hilbert de las wavelets básicas:

$$
H \theta_{j, l}(t)=\sum_{k} \mu_{m k} F_{m}(l, k) H \psi_{j, n+k}(t),
$$

lo cual amplía los horizontes del análisis, [8].

Queda abierta en esta dirección la posibilidad de generar clases más amplias de funciones elementales, en particular, paquetes con formas de onda más largas y espectros a través de las escalas, del tipo chirplets, en el contexto de análisis multirresolución. Estas atractivas propuestas aumentarían la potencia operativa de las wavelets. 


\section{Conclusiones}

En esta exposición hemos presentado las contribuciones de la transformada wavelet al análisis tiempo-frecuencia. Los métodos expuestos permiten realizar en el contexto de un único esquema, el análisis multirresolución, eficientes técnicas de representación tiempo-frecuencia, en cierto sentido, combinando y englobando diversas ténicas.

De esta forma, es posible implementar estrategias flexibles adaptadas a las características de la señal y al problema bajo consideración.

Quedan abiertas otras alternativas y promisorias líneas de desarrollo capaces de explotar todo el potencial de las wavelets.

\section{Referencias}

[1] Cohen, L. (1995) Time-Frequency Analysis. Prentice Hall Signal Processing Series, New Jersey.

[2] Jaffard, S.; Meyer, Y.; Ryan, R. (2003) Wavelets: Tools for Science and Tecnology. SIAM, Philadelphia.

[3] Gröehenig, K. (2001) Foundations of Time-Frequency Analysis. Birkhäuser, Boston.

[4] Huang N.E. et al. (1998) "The empirical mode decomposition and the Hilbert spectrum for non-stationary tyme series analysis", Proc. R. Soc. A, Mathematical, Physical \& Engineering Sciences 454(1971): 903-995.

[5] Mallat, S. (2009) A Wavelet Tour of Signal Processing, The Sparse Way. Academic Press, San Diego.

[6] Meyer, Y. (2001) Oscillating Pattern in Image Processing and Nonlinear Evolution Equations. American Mathematical Society, Providence RI.

[7] Serrano, E.; Figliola A.; Fabio, M. (2009) "Diseño de Marcos de Paquetes de Wavelets", XIII RPIC Reunión de Trabajo en Procesamiento de la Información y Control, 16-18 Sep 2009, Rosario, Argentina.

[8] Serrano, E.; Aragón, A. (2009) "Métodos numéricos basados en onditas para el cálculo de la Transformada de Hilbert", XIII RPIC Reunión de Trabajo en Procesamiento de la Información y Control, 16-18 Sep 2009, Rosario, Argentina.

[9] Walnut, D.F. (2003) An Introduction to Wavelet Analysis. Birkhäuser, Boston. 